\title{
Territorialisation sanitaire et décentralisation : état des lieux et enjeux à partir du cas français
}

Emmanuel Eliot, Véronique Lucas-Gabrielli et Catherine Mangeney

\section{OpenEdition}

Journals

Édition électronique

URL : http://journals.openedition.org/rfst/577

DOI : $10.4000 /$ rfst.577

ISSN : 2492-3672

Éditeur

Espaces et SOciétés (UMR 6590)

Référence électronique

Emmanuel Eliot, Véronique Lucas-Gabrielli et Catherine Mangeney, «Territorialisation sanitaire et décentralisation : état des lieux et enjeux à partir du cas français », Revue francophone sur la santé et les territoires [En ligne], Décentralisation et territorialisation sanitaire, mis en ligne le 06 juin 2017, consulté le 06 avril 2021. URL : http://journals.openedition.org/rfst/577 ; DOI : https://doi.org/ $10.4000 /$ rfst. 577

Ce document a été généré automatiquement le 6 avril 2021.

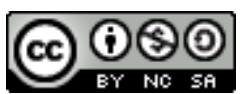

La Revue francophone sur la santé et les territoires est mise à disposition selon les termes de la Licence Creative Commons Attribution - Pas d'Utilisation Commerciale - Partage dans les Mêmes Conditions 4.0 International. 


\title{
Territorialisation sanitaire et décentralisation : état des lieux et enjeux à partir du cas français
}

\author{
Emmanuel Eliot, Véronique Lucas-Gabrielli et Catherine Mangeney
}

\section{Introduction}

1 Dans un très grand nombre de pays dans le Monde, les réformes destinées à accroitre l'efficience des systèmes de santé furent amorcées dans les années 1970 puis renforcées dès le début des années 1990. Elles s'inscrivent dans un processus général de décentralisation des institutions auquel est associé une montée en puissance du «local» comme espace d'action. Cette territorialisation prend des formes diverses en raison de l'héritage des systèmes de santé en place, de leurs modes d'organisation ou de choix opérés par les acteurs. Cependant, il existe des points communs du point de vue des politiques conduites depuis plus de vingt ans: valorisation des mailles d'action fines et des espaces considérés comme étant auparavant marginalisés, discours normatif sur la proximité considérée comme la solution pour pallier les inégalités de santé, diffusion de modèles de gestion issus du "New Public Management 1 ", multiplication des intermédiaires dans l'organisation et l'activité de soin.

2 A la suite du séminaire organisé en novembre 2015 à Paris dans le cadre de l'axe « Santé et territoires » du collège International des sciences du territoire (CIST) à l'institut d'aménagement et d'urbanisme d'île-de-France, cet article propose d'introduire le dossier «territorialisation et décentralisation sanitaire ». Dans un premier temps, il s'agira de préciser les logiques qui sous-tendent les recompositions en cours des systèmes de santé dans le monde et qui sont au cœur de la phase actuelle de territorialisation des systèmes de soins. Dans une perspective géographique et d'aménagement, une proposition de définition de cette territorialisation s'appuyant à partir de l'analyse des instruments de l'action publique (Lascoumes, Simard, 2011) sera esquissée. Dans un deuxième temps, il s'agira de préciser à partir de l'exemple français 
comment ces différents instruments (lois, outils...) ont fait évoluer la territorialisation sanitaire depuis la deuxième Guerre Mondiale. Enfin, dans un troisième temps et au regard de l'exemple français, les enjeux contemporains et les problèmes associés à la phase actuelle de territorialisation seront discutés.

\section{Les tendances de la recomposition des systèmes de soins dans le monde}

\section{Trois logiques complementaires}

3 A la suite de la construction et de la mise en place de systèmes de santé fondés sur l'accès aux soins pour tous aux lendemains de la deuxième Guerre Mondiale dans de nombreux pays du monde, la crise économique, la modification des équilibres démographiques et les nouveaux enjeux sanitaires à partir des années 1980, ont montré les difficultés d'adaptation des systèmes à l'évolution du contexte dans lequel ils s'inscrivaient, conduisant à une recomposition régulière des systèmes de soins qui s'est intensifiée au cours des années 2000. Aujourd'hui, la plupart des pays du monde sont concernés par ces transformations qui reposent sur trois facteurs: la régulation financière, le développement des partenariats et le transfert des compétences.

4 Premièrement, la logique financière a été placée au centre de l'évolution et de la viabilité des systèmes de soins. Elle a généralement consisté à modifier les modalités d'allocation des ressources ou à opérer une plus grande sélection des interventions et actions à financer, engendrant des problèmes d'arbitrage entre qualité des soins et dépenses. Face à ces difficultés, différents types de régulation ont été conduits : gestion des coûts, réforme des financements, allocation des budgets en fonction des besoins, évaluation de la qualité rendue sur des bases de contrôles statistiques, développement de regroupements, mutualisation et concurrences dans l'allocation des budgets... La littérature interroge les inégalités produites par ces changements (Arredondo et alii, 2005 en Amérique du Sud; Curtis, Leonardi, 2012 en Suède et au Royaume-Uni; Wilensky, 2012 aux Etats-Unis) ainsi que le thème de l'équité dans l'allocation des ressources (Lane et alii, 2017). Elle met l'accent sur la diversités des dispositifs vis-à-vis des marchés mis en place selon les pays (Giaimo, Manow, 1999) en Allemagne, Royaume-Uni et Etats-Unis ; Hughes et alii., 2010 en Thaïlande) et sur la nécessité d'une coordination entre Etats et types de financeurs (Bowser et alii, 2014 pour le cas des financements de santé mondiaux ; Bossert, Mitchell, 2011 au Pakistan).

5 Deuxièmement, les partenariats ont été encouragés ou sont devenus obligatoires entre les acteurs publics, privés ou issus de la société civile. Ces modes relationnels ont généralement pris la forme d'une contractualisation d'objectifs et/ou de moyens destinés à " optimiser » la performance des systèmes de soins. La mise en place de ces contrats est toutefois variable selon les pays et répond le plus souvent à des objectifs d'accessibilité aux équipements et services. Certains ont introduit des coopérations entre les établissements de soins (Brown, Barnett., 2004, en Australie), d'autres ont généralisé la contractualisation en cherchant à intégrer l'ensemble des acteurs de la hiérarchie sanitaire. Le plus souvent, cette contractualisation répond à un objectif managérial et vise à développer l'accès à des services ou des plateaux techniques inexistants auparavant. La forme la plus développée de ces coopérations est le Partenariat Public Privé (PPP). Portés par les politiques de l'Organisation Mondiale de 
la Santé et de la Banque Mondiale, les PPP ont été érigés comme symboles du renouveau d'une gouvernance sanitaire mondiale (Guilbaud,2015). Ces dispositifs visent différents objectifs à différentes échelles: par exemple, le développement d'une offre de soins au sein d'un territoire municipal ${ }^{2}$ ou le financement - via un consortium de banques et de promoteurs du Bâtiment et Travaux Publics - pour la création de nouveaux établissements hospitaliers à l'échelle d'un Etat par les autorités publiques ${ }^{3}$ (Pollock, Price, 2014). Au niveau international, les partenariats Publics Privés Sanitaires Internationaux (PPSI) ont avant tout orientés leurs actions vers l'accès aux médicaments dans le cadre des grands programmes d'action de l'Organisation Mondiale de la Santé, impliquant aussi des entreprises et des fondations. Cela étant, si l'usage du terme de PPP est très fréquent, le dispositif au sens strict n'est pas majoritaire (Raman, Björkman, 2015). Le terme recouvre en effet d'une part, un très grand nombre de formes de contractualisation entre des acteurs des sphères privées et publiques mais aussi du monde associatif, d'Organisation Non Gouvernementales, voire dans certains pays de mouvements religieux, et d'autre part, des types de dispositifs très divers (gestionnaires, économiques, financiers...) qui ne permettent, le plus souvent, pas de développer une offre de soins complète.

6 Troisièmement, ces réformes structurelles ont été accompagnées de cadres législatifs destinés à développer ou renforcer le niveau local de l'action publique en opérant un transfert de pouvoirs de l'Etat à des relais. Cependant, les modalités du transfert sont différentes, allant de la décentralisation à la déconcentration, en passant par la dévolution et la délégation, voire même à la privatisation (Polton, 2004). En Côte d'Ivoire, par exemple, la politique de communalisation amorcée dans les années 1980 s'est renforcée depuis 2011 avec la création de districts et des conseils généraux. Ces entités décentralisées possèdent une certaine autonomie proche d'une dévolution mais elles ne jouent cependant pas de rôle dans la planification sanitaire qui reste aux mains du pouvoir central (Soura, Coulibaly, 2014). Plusieurs types de décentralisation peuvent aussi coexister dans un même territoire national. En Inde, par exemple, dans le cadre du programme rural de développement sanitaire lancé en 2005 (National Rural Health Mission) dans les Etats les plus pauvres, une série de mesures à chercher à renforcer la dévolution en augmentant la capacité des institutions locales (Panchayat Raj) et en créant de nouveaux représentants sanitaires communautaires (Asha) afin de planifier, organiser et assurer les services de soins ${ }^{4}$. En même temps, à l'échelle régionale, le programme RSBY (Rashtryia Swasthya Bima Yoajana) est destiné à fournir une offre de santé à des populations en dessous du seuil de pauvreté à travers des réseaux de soins identifiés (Rajasekhar et alii, 2011). Reposant sur une délégation de services à des assurances privées et proposant une offre tant privée que publique, le programme s'est étendu à une grande partie des Etats et des Territoires de l'Union depuis son lancement en 2007.

Dans tous les cas, le pouvoir devient plus diffus dans les territoires. Il ne dépend plus de uniquement de la taille des institutions mais du degré de pouvoir et de la capacité des acteurs à l'activer. Le développement de ces relais fait ainsi émerger de nouveaux enjeux : coût de ces agencements et organisations, conflits potentiels ou réels entre différents types d'acteurs (Arora, et alii, 2000,à Londres), entre entités administratives (Blanchet, 2016, dans le cas de la gérontologie en France par exemple), entre gouvernements centraux et locaux (Frederiksson et alii, 2012, en Suède), dédoublement des activités, besoins de coordination et de mise en synergie entre des acteurs avec des 
rôles divers (financeurs, gestionnaires, soignants...), (Lewis., 2008, en Australie, par exemple).

\section{Comment définir la territorialisation en cours ?}

Les transformations contemporaines des systèmes de soins présentent en somme de nouvelles constructions qui produisent une image complexe des paysages sanitaires. Elles peuvent certes générer de nouvelles coopérations, modifier les hiérarchies anciennes, réorganiser les périmètres institutionnels mais elles peuvent aussi, en contrepartie, renforcer certaines inégalités en termes d'offre ou d'accès et compartimenter le rôle des acteurs. En inscrivant plus fortement l'action au niveau local, tout en l'insérant dans une diversité de réseaux le dépassant, les recompositions en cours des paysages sanitaires nécessitent de redéfinir les échelles d'action et de modifier les manières d'organiser les configurations des systèmes de soins.

La territorialisation qualifie ces évolutions générales et fait l'objet d'une utilisation plurielle. Même si, à la différence du terme de territoire, il met l'accent sur un processus (Amat-Roze, 2011), il définit d'une part, l'analyse de la diversité des modes d'appropriation (symboliques, économiques, sociaux...) des espaces et d'autre part, les programmes de l'action publique reposant sur le décloisonnement des secteurs d'activités et visant à introduire une diversité dans les modes de production des espaces aménagés. Dans la littérature anglophone traitant des systèmes de soins, le terme de territorialisation (territorialization) est souvent associé aux réflexions sur l'évolution de l'offre sanitaire dans un contexte de néolibéralisme (Prince, et alii, 2006; Murray et alii, 2016, par exemple). Le terme qualifie ces transformations au regard des jeux de pouvoir qu'elles mettent en jeu. Dans la littérature géographique francophone traitant des systèmes de soins, la territorialisation est employée pour prendre en compte les modalités d'adaptation d'une offre de service à l'espace socialement construit par les acteurs (Fleuret, 2016) ayant des effets positifs ou négatifs sur la santé des habitants, et pour identifier les mécanismes permettant d'ancrer une politique dans la production du territoire afin d'améliorer une situation sanitaire (Rican , Vaillant, 2009).

En raison de la diversité des dispositifs et des types d'action conduits par les acteurs, l'enjeu est probablement de mieux comprendre les modes opératoires de cette territorialisation et d'interroger la diversité des formes territoriales produites. Dans cette perspective, on peut chercher à caractériser et à comprendre les transformations en cours sous l'angle des instruments de l'action publique (Halpern et alii, 2014) qui produisent des normes en matière de distances et de niveaux d'action. Les instruments permettent en effet d'appréhender les caractéristiques globales et locales du système de soins en se plaçant au niveau des intermédiaires opérant la transformation des paysages sanitaires (Eliot, 2016). Dans cette perspective, l'instrument est un dispositif technique porteur d'un mode de spatialisation spécifique qui répond à un objectif de régulation. De cette manière, la territorialisation en cours dans les différents pays permet de prendre en compte les questions du pouvoir et des ressources. Pouvoir au sens large, des institutions ou de l'Etat, mais également des groupes et communautés, avec leurs acteurs et leurs modes de relations; ressources "effectives " en termes d'offre de soins, de dispositifs de gestion, de modes d'accès mais aussi ressources "potentielles »: financières, structurelles, sociales, technologiques... destinés à 
développer les ressources effectives. En effet, les instruments permettent de mettre en jeu les ressources destinées à déployer des actions sanitaires mais sont aussi des intermédiaires du pouvoir. Ainsi, en positionnant les instruments au cœur de l'analyse, la territorialisation se définie à l'interface d'une part, de la gouvernance - en tant que processus de coordination d'acteurs et d'institutions -, d'autre part, du gouvernement comme capacité des acteurs à imposer des choix en fonction de contraintes délimitant les périmètres d'action des acteurs -, et, enfin, des pratiques sociales organisant l'action en fonction de représentations et significations. Ces différents agencements de métriques et de niveaux déployés via les instruments produisent une grande diversité de formes territoriales selon les lieux.

Figure 1 : La territorialisation : essai de définition

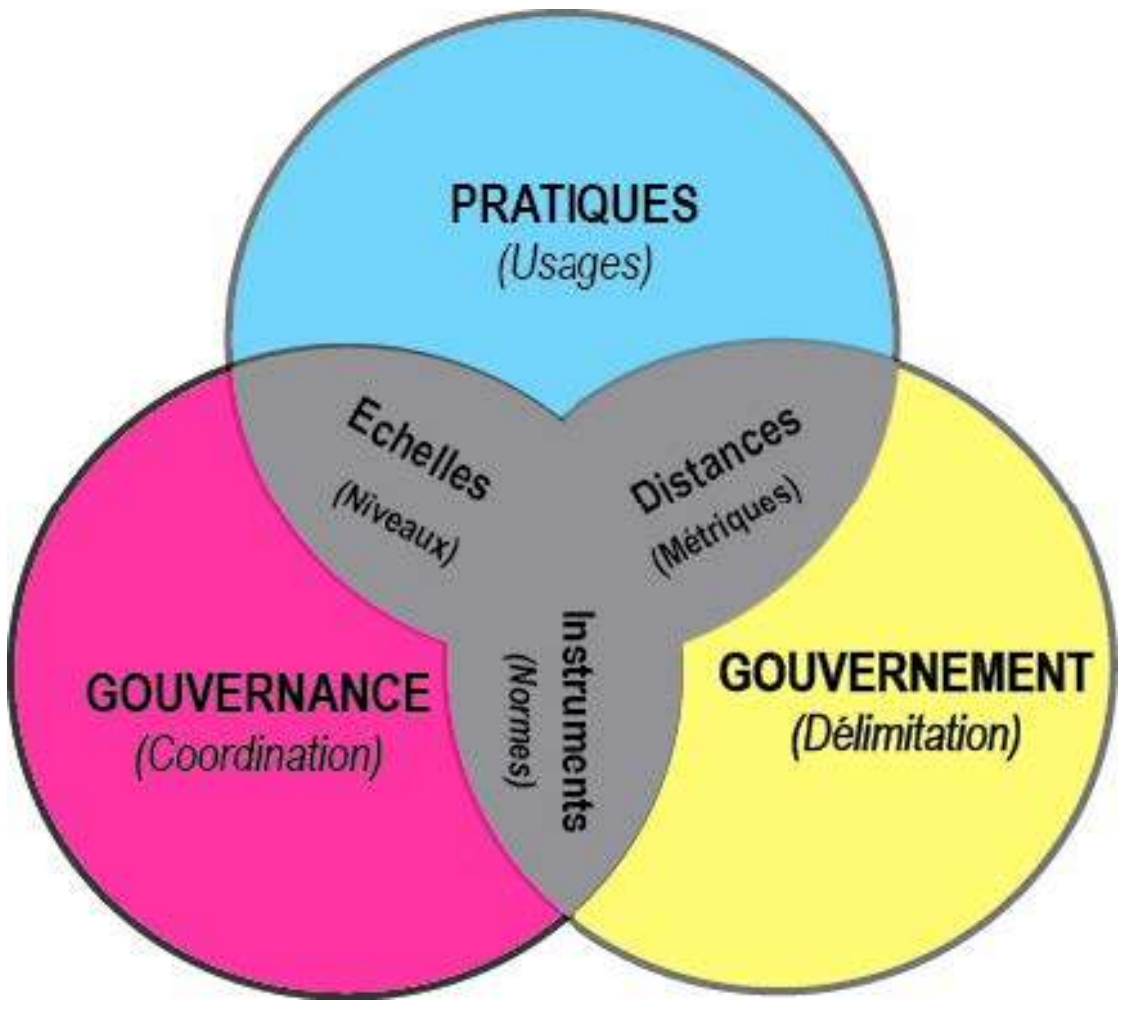

\section{Le cas français : décentralisation territoriale et décentralisation fonctionnelle en santé, quelle articulation?}

11 L'analyse de la décentralisation dans le domaine de la santé est ici appréhendée en proposant au préalable une description générale de la décentralisation des institutions en France afin de pouvoir mettre en exergue la manière dont ces deux processus se sont articulés.

\section{L'évolution du « mille feuilles » territorial}

Après la deuxième Guerre Mondiale, le développement du territoire français est caractérisé par un profond déséquilibre entre régions (Gravier, 1947). Pour faire 
contrepoids à l'hypertrophie de la région parisienne, le décret du 30 juin 1955 crée vingt et une régions économiques de programme. L'évolution de ces dernières sera ensuite très progressive : transformation en «circonscriptions d'action régionale » de l'État en 1959, ajout de structures administratives et consultatives en 1963, acquisition du statut «d'établissement public régional» doté d'un conseil composé d'élus (parlementaires et élus locaux) en 1972. C'est finalement la loi de décentralisation du 2 mars 1982 qui transforme les régions en collectivités locales de plein exercice, même s'il faudra attendre 1986 pour voir leurs assemblées élues au suffrage universel direct.

13 Ce qui sera rétrospectivement présenté comme l'acte I de la décentralisation rassemble des évolutions dont la ligne directrice est le critère démocratique, compris comme requérant le rapprochement de la décision publique du citoyen et se traduisant par des capacités d'actions accrues pour les collectivités locales. Le design de la décentralisation française des années 1980 - où la décentralisation fiscale (attribution d'un pouvoir de vote des taux d'imposition locale dès 1980) précède la décentralisation politique (suppression en 1982 de la tutelle a priori et de la fonction exécutive régionale et départementale confiée jusque-là au préfet) qui précède elle-même la décentralisation administrative (transferts de compétences) - exprime une volonté de renforcer le pouvoir et l'autonomie des élus locaux vis-à-vis de l'Etat (Le Lidec, 2011).

14 L'acte II de la décentralisation (2003-2004), lui, s'inscrit plus dans une logique de décentralisation administrative renforçant compétences et responsabilité des élus locaux sans accroître leur autonomie politique ni leur capacité à faire varier leurs revenus. Alors qu'une loi organique, en 2004, vient préciser les conditions de l'autonomie financière des collectivités territoriales, l'érosion de leurs marges de manœuvre budgétaires est amorcée. Même si des blocs de compétences commencent à se dessiner - développement économique et formation professionnelle pour les régions, action sociale pour les départements - les critères des transferts illustrent avant tout la volonté propre de l'État et la recherche d'équilibres entre les différents niveaux de collectivités - transfert de l'essentiel du réseau routier national et des personnels techniques et de services de l'éducation nationale.

15 Ce n'est qu'avec l'acte III, qui regroupe formellement les lois adoptées entre 2013 et 2015 mais auquel il faut rattacher, dans l'esprit, la loi « de réforme des collectivités territoriales » de 2010, qu'est affirmée véritablement une vision structurée de la décentralisation. C'est d'abord le cas avec le renforcement de la spécialisation des différents niveaux dans une logique de subsidiarité ou, à tout le moins, de recherche du bon niveau d'intervention. En ce sens est supprimée la clause de compétence générale (capacité à intervenir dans tous les champs) des régions et départements. Du point de vue de leurs attributions, même si les transferts sont en fin de compte limités, les premières sont renforcées au détriment des seconds (développement économiques, transports non urbains, planification). Leur faculté à contribuer en commun aux projets locaux (financements croisés) est très strictement encadrée.

16 Dans le même temps, la réorganisation de la décentralisation se traduit par un impératif renforcé d'efficacité, qui vise prioritairement les communes, lesquelles sont nombreuses et à l'origine de la part la plus importante de l'investissement public en France. Ainsi, aux incitations financières au regroupement intercommunal de la décennie 2000 succède, dans les années 2010, la généralisation obligatoire de l'adhésion à un établissement public de coopération intercommunale. Ceux-ci sont désormais toujours plus grands (au moins 15000 habitants et parfois plus d'une centaine de 
communes), plus intégrés en terme de compétences (l'exemple le plus abouti étant celui des Métropoles qui exercent la quasi-totalité des attributions locales et empiètent même progressivement sur les compétences départementales), dotés d'une fiscalité propre et d'élus désignés par le suffrage universel direct. Aux mutualisations s'ajoute une pression accentuée de l'État sur les ressources locales, notamment via la diminution progressive de la dotation globale de fonctionnement.

17 En parallèle, les ferments d'une évolution plus profonde de la décentralisation française se font jour. C'est le cas avec l'affirmation systématique de la responsabilité de coordination de la région à travers ses plans et schémas. L'ampleur des domaines traités et surtout leur portée prescriptive toujours plus importante enfonce deux coins dans la conception unitaire de la République française: une forme possible de différenciation de la norme sur le territoire et, non pas une tutelle, mais une réelle capacité d'orientation de la région sur les autres niveaux. Le complément en termes de gouvernance se profile avec la montée en puissance possible des Conférences territoriales de l'action publique, instances de dialogue entre collectivités, présidées par les régions et dédiées à l'ajustement des compétences, délégations, et modalités d'action commune aux spécificités des différentes configurations locales.

\section{Une territorialisation sanitaire incomplète}

La santé est restée à l'écart des nombreux transferts législatifs. Les collectivités ne se sont vues attribuer par exemple qu'un rôle consultatif dans les instances (dans le conseil de surveillance des Agences Régionales de Santé (ARS)) ou bien un rôle d'intervention par des mécanismes de délégation de compétences dans certains domaines comme les programmes de prévention et de vaccination.

Pourtant la régulation du système de soins au niveau régional est pensée depuis longtemps en France. Le VI ème plan (1971-1975) est le signe d'un véritable tournant dans l'état d'esprit du planificateur. En présentant une réflexion globale sur le système de santé axée sur des objectifs et des actions prioritaires, il propose une vision nouvelle de la planification (Henrard, 1995). Concomitamment, la loi du 31 décembre 1970 définit pour la première fois les conditions d'une planification volontariste de l'espace (Brau, Vigneron, 1996). Pour ce faire, elle instaure le service hospitalier public et la carte sanitaire qui subordonne la création ou l'extension d'établissements en lits ou équipements lourds aux besoins sur la base de ratios d'équipement définis au niveau national. Arrêtée en 1974, ce découpage de l'espace géographique aboutit à la définition de 256 secteurs sanitaires infra départementaux et de taille variable (Amat-Roze, 2011) dont la construction repose essentiellement sur les aires d'attraction urbaine puis sur l'attraction des établissements de soins. Ce premier outil de planification sanitaire n'en reste pas moins très rigide et quantitatif car il vise davantage les principes d'égalité que d'équité (Coldefy, Lucas, 2012). La logique de planification par plan est progressivement abandonnée. Vingt ans plus tard, la loi hospitalière de 1991consacre l'échelon régional comme le niveau structurant de la politique hospitalière en créant les schémas régionaux d'organisation sanitaire (SROS). Les SROS précisent la manière dont les soins doivent être organisés pour répondre de manière adaptée aux besoins localisés des populations. Il n'y a plus comme auparavant d'objectifs uniformes appliqués dans chaque région mais une prise en compte des spécificités locales devant conduire à une meilleure adaptation de l'offre de soins (Coldefy, Lucas, 2012). Cette période est 
l'occasion pour certaines régions d'associer des géographes de la santé à leur réflexion. Dans cette dynamique, les ordonnances d'avril 1996 instaurent de nouvelles instances régionales dont les ARH (agences régionales d'hospitalisation) qui pilotent la planification des structures hospitalières publiques et privées tandis que les URCAM (unions régionales des caisses d'assurance maladie) coordonnent l'action des caisses et développent la politique de gestion du risque régionale. L'ordonnance du 4 Septembre 2003 supprime la carte sanitaire et fait du SROS l'unique outil de planification. Le territoire n'est plus envisagé comme un périmètre administratif mais comme un lieu d'organisation des soins encadré par des objectifs quantifiés de l'offre de soins.

La loi du 21 juillet 2009 portant réforme de l'hôpital et relative aux patients, à la santé et aux territoires, dite loi HPST, est une nouvelle étape car elle simplifie l'organisation jusque-là éclatée de la politique sanitaire régionale en rassemblant au sein de nouvelles agences régionales de santé les pouvoirs des ARH, des directions régionales et départementales des affaires sanitaires et sociales (Drass et Ddass), des URCAM, des groupements régionaux de santé publique et des caisses régionales d'assurance maladie (Cram). Les ARS correspondent à une déconcentration un peu particulière. Certes, il s'agit d'un aménagement de l'action de l'Etat et non d'une dévolution de compétences aux collectivités territoriales mais l'ARS est une institution ayant la personnalité morale, correspondant plutôt aux principes d'une décentralisation fonctionnelle (Combeau, 2012) dans laquelle l'établissement public est soumis à la surveillance et à l'orientation de l'Etat.

21 Cette nouvelle organisation rend possible le décloisonnement des politiques régionales hospitalières, ambulatoires et médico-sociales. Celle-ci est mise en œuvre dans le plan régional de santé (PRS) qui définit, en cohérence avec la stratégie nationale de santé, les objectifs pluriannuels de l'ARS dans ses domaines de compétence, ainsi que les mesures pour les atteindre. Pour le déployer, les ARS ont développé une politique de contractualisation et de partenariat avec de multiples acteurs dont les associations et les collectivités territoriales (Schapman-Ségalié, Lombrail, 2013) qui deviennent des acteurs privilégiés pour décliner le PRS au niveau local mais aussi des interlocuteurs incontournables pour faire émerger une connaissance de l'intérieur des lieux (Rican, Vaillant, 2009) permettant de mieux intégrer les spécificités locales dans les politiques régionales. Les associations sont ainsi très présentes dans certains secteurs (handicap, aide à domicile) où elles jouent le rôle de gestionnaire de service et comblent parfois localement les vides du système de santé (Fleuret, 2006). Les collectivités territoriales, malgré l'absence de compétences légales dans le domaine sanitaire, sont quant à elles largement impliquées dans ce nouveau système de santé régional, qui fait de la territorialisation, du décloisonnement, de la conception globale de la santé, de la fluidité des parcours de santé et de la réduction des inégalités des axes majeurs de son action. Cette implication est d'autant plus forte qu'elle répond aux sollicitations des citoyens qui sont telles que nombre de maires font de la lutte contre la désertification médicale de leurs territoires une de leur priorité. Enjeu politique, la santé devient un enjeu pour les politiques et un domaine d'action pour les collectivités au travers de la résolution pragmatique de problèmes locaux (Monneraud, 2012). Cette municipalisation de fait (et non de droit) de la santé publique conduit à des situations et implications hétérogènes (Vaguet, 2011). Pour autant, les collectivités se trouvent confrontées à l'effet de ciseaux entre la demande sociale qui leur est adressée et des finances qui se tendent. Leur capacité à faire, et ce, en fonction des besoins de leurs 
habitants, reste un sujet à part entière des débats actuels autour des finances locales et des systèmes de péréquation.

\section{Les enjeux en France aujourd'hui}

\section{Des formes territoriales à repenser et réorganiser}

Ainsi, déconcentration et décentralisation se développent en parallèle ou conjointement, générant chevauchements, financements croisés et relative illisibilité de l'organisation administrative sur l'ensemble du territoire (Kada, 2012). La place de l'Etat et des territoires dans l'action publique ne cessent d'être redéfinis dans de nombreux domaines d'action publique (Aust \& Cret, 2012), l'Etat devenant un acteur parmi d'autres des politiques publiques. La recomposition de la gouvernance sanitaire ne fait qu'écho à ce que l'on observe dans d'autres domaines d'action publique (enseignement et recherche, développement durable, gestion de l'eau et des déchets, aménagement $d u$ territoire, politiques sociales,...). Lors du séminaire, Luc Ginot présentait la position de l'ARS Île-de-France à l'interface de ses missions de régulation et d'organisation, garante de l'équité territoriale et dotée de compétences larges et d'outils de territorialisation, devant composer avec les autres services territorialisés de l'Etat, avec l'Assurance Maladie, avec les territoires et les élus (démocratie sanitaire, CLS,...) et à qui échappent de nombreux leviers d'action et de régulation directs (compétences des collectivités, indépendance du système libéral,...).

Ainsi, face à la montée des politiques de développement local, de spatialisation de la décision publique (Méasson, 2008) mais aussi des principes de gouvernance territoriale, de démocratie partici-pative et des dispositifs de concertation (Torre 2015), les modes d'action publique doivent se réinventer, entre hiérarchie, contrainte ou incitations, concurrence par l'allocation des fonds étatiques, contractualisation et recherche d'une co-construction de l'action publique, avec une prévalence accrue de la coordination entre acteurs de natures différentes (productifs, associatifs, particuliers, représentants des pouvoirs publics ou des collectivités locales...) et niveaux multiples ${ }^{5}$. Les réponses sont variables d'un territoire à l'autre et dépendent de la configuration spécifique de chaque territoire. Ce dernier apparaît alors comme une entité active qui puise son potentiel de développement de ses ressources locales et des jeux d'associations, de concertations, de négociations ou de conflits qui les animent (Torre, op. cit .). Pour le secteur associatif notamment, se posent alors des enjeux forts de visibilité, de résilience et de pérennité d'action face aux financements mais aussi aux temporalités qui se raccourcissent, dans un calendrier bien souvent subi (Fleuret \& Skinner, 2010; Nahapétian, 2012).

24 Cette mutation de l'action publique et le développement des politiques territoriales multi-niveaux, de l'inter-territorialité ou du «local multiterritorial» (Vanier, 2015), conduisent au développement de "coopération souples » d'échelles méso-territoriales prenant la forme de partenariats peu formalisées, flexibles et volontaires de dialogue entre acteurs locaux (Parnaix et Zugetta, 2015) ou de «territoires de projet » de formes plus ou moins institutionnalisées, supposant la quête de la bonne "mosaïque territoriale » (Beauchard, 2003) et de la meilleure façon de gérer celle-ci, inscrivant la centralité transactionnelle dans un espace bien plus large que celui du territoire. 
25 Le projet commun et la coordination / coopération des acteurs deviennent alors les maîtres mots des relations qui caractérisent la nouvelle configuration du système de soins (Sabi, 2016), mais plus globalement du système de gouvernance territoriale.

\section{Deux grands enjeux}

\section{Le développement de projets portés par des coalitions d'acteurs}

26 Si l'échelon local (dont la définition des contours reste éminemment floue) et ses interfaces s'imposent de manière croissante comme le niveau optimal de mise en œuvre de l'action publique et d'innovation, sa concrétisation par " projets » ne peut se faire sans que se dégage une "coalition» d'acteurs (publics, privés, associatifs, d'usagers) portant ou soutenant le projet, coalition qui ne peut exister sans langage et objectifs communs, sans concertation solide, sans convergence minimale d'intérêts, en reconstruction permanente à la fois dans l'espace et le temps (Bourdin, 2003). Il s'agit également de se réadapter sans cesse aux nouvelles dynamiques d'acteurs qui se forgent au gré des recompositions territoriales, de la refonte des compétences, des marges de manœuvre budgétaires, des alternances politiques, de la création de nouvelles instances et des nouvelles réglementations.

\section{Coordinations, coopération et complémentarités}

27 Dans ce contexte, la coordination des acteurs et des « lieux» devient incontournable. Cette coordination, plus ou moins formalisée, vise à créer, de surcroît, les conditions d'une coopération et d'une complémentarité entre les acteurs et les différentes actions mises en œuvre (Sabi, op. cit .).

28 Dans le champ sanitaire, cette recomposition organisationnelle se caractérise aujourd'hui par le développement de structures hybrides de soins coordonnées, la création de réseaux de soins ville-hôpital, le développement des accords de partenariats entre structures et instances sanitaires ou non sanitaires et l'accroissement des coopérations entre secteur privé et secteur public.

29 Cette coordination prend des formes fort variées, ascendantes ou descendantes, coercitives ou incitatives : protocolisation des soins, règles, normes, bonnes pratiques, contractualisation, conventions, incitations, dotations et allocations de ressources, délégation, mais aussi coopérations "spontanées" basées sur la confiance interindividuelles. Les innovations technologiques et les potentialités qu'elles offrent ouvrent encore la voie à des nouvelles modalités organisationnelles. La puissance publique est alors en quête sans cesse renouvelée de nouveaux cadres facilitant ces coopérations et coordinations, dans une vision d'efficience autant que d'égalité des chances: groupements hospitaliers de territoire, maisons de santé pluriprofessionnelles, rémunération de la coordination, contrat territorial de santé,... avec des effets parfois limités et une adhésion des acteurs pouvant s'avérer conflictuelle.

\section{Conclusion}

Ainsi, dans un très grand nombre de pays du monde, la décentralisation - associée à une montée en puissance $\mathrm{du}$ «local» comme espace d'action - constitue un des axes 
clés des réformes des systèmes de santé conduites depuis le début des années 1990. Considérée comme un levier pour renforcer l'efficacité, l'efficience et la performance des systèmes, elle a été généralement associée à un transfert de compétences, de responsabilités et de fonctions à des collectivités locales ainsi qu'à un ensemble élargi d'acteurs (publics, privés, associatifs...), la logique de contractualisation accompagnant ou supplantant la logique de planification, et bousculant les systèmes d'acteurs et les temporalités. Envisagée comme favorisant la participation citoyenne et la mise en adéquation entre l'offre et les besoins de santé, la territorialisation des questions de santé contribue à faire évoluer les espaces sanitaires en produisant de nouveaux découpages et en ciblant les actions de santé auprès des populations dans des lieux ainsi qu'en impulsant de nouveaux modes de régulation et de gouvernance des espaces. Cette mutation des échelles spatiales, temporelles et organisationnelles dans le domaine de la santé se retrouve dans les autres champs de l'action publique, complexifiant le système territorial (Moine, 2006), l'articulation entre le jeu des échelles spatiales et organisationnelles, et bouleversant la stabilité des systèmes d'acteurs. L'acte III de la décentralisation en France rebat actuellement les cartes de la gouvernance, du financement, de l'organisation et des compétences des collectivités territoriales, instaure les métropoles et la notion d'intérêt métropolitain. Ces évolutions réinterrogent une nouvelle fois les modes de faire ensemble localement et de manière transversale, et ce d'autant plus fortement que la santé tend à s'appréhender aujourd'hui de manière " globale ».

\section{BIBLIOGRAPHIE}

Amat-Roze J-M., 2011, « La territorialisation de la santé : quand le territoire fait débat », in Hérodote , 4, 143, pp. 13-32.

Arora S., Davies A., Thompson S., 2000, « Developing health improvement programmes: challenges for a new millennium » in Journal of Interprofessional Care, 14, 1, pp. 9-18.

Arredondo A., Orozco E., Deicaza E., 2005, « Evidences on weaknesses and strengths from health financing after decentralization: lessons from Latin American countries » in The International Journal of Health Planning and Management, 20, 2, pp., 181-204.

Aust J., Cret B., 2012, L'Etat entre retrait et réinvestissement des territoires, Revue française de sociologie 2012/1, vol. 53, p.3-33.

Beauchard J., 2003, Territoire et identités, in Beauchard J. (dir.), La mosaïque territoriale, enjeux identitaires de la décentralisation, Paris : éditions de l'Aube, Bibliothèque des territoires, pp. 7-18.

Blanchet M., 2016, « Regards croisés d'acteurs gérontologiques territorialisés sur la loi Hôpital Patients Santé et Territoires (HPST) » in Annales de géographie, 2, 708, pp. 170-92.

Bossert T., Mitchell A., 2011, « Health sector decentralization and local decision-making: Decision space, institutional capacities and accountability in Pakistan ». In Social science medicine , 72,1, pp. $39-48$. 
Bowser D., Sparkes S.P., Mitchell A, Bossert T.J., Bärnighausen T., Gedik G., Atun R., 2014, « Global Fund Investments in Human Resources for Health: Innovation and Missed Opportunities for Health Systems Strengthening » In Health Policy and Planning, 29, pp. 986-97.

Bourdin A., 2003, Gouvernance du « vivre ensemble » et gouvernance du projet, in Beauchard J. (dir.), op. cit .

Vigneron E., Brau F., 1996, « Approches géographiques de la planification sanitaire : concepts et méthodes », in Cahiers Geos, 32, pp. 1-4.

Brown L., Barnett J.R., 2004, « Case study: Is the corporate transformation of hospitals creating a new hybrid health care space? A case study of the impact of co-location of public and private hospitals in Australia » in Social Science and Medicine, 58, pp. 427-44.

Chappoz Y., Pupion P-C., 2012, « Le new Public Management » In Gestion et management Public, 1, 2, pp. 1-3.

Coldefy M., Lucas Gabrielli V., 2012, Le territoire, un outil d'organisation des soins et des politiques de santé : évolution de 2003 à 2011. Questions d'Economie de la Santé, $\mathrm{n}^{\circ} 175$.

Combeau P., 2012, Territorialisation de la politique de santé en France, in Castaing C. (dir.), La territorialisation des politiques de santé, Bordeaux : Les Etudes Hospitalières, Collection Actes et séminaires, p. 35-44.

Curtis S., Leonardi G., 2012, Health, wealth and ways of life: what can we learn from the Swedsih, US and UK experience? Introduction to the commentaries, Social science and Medicine, 74 (5), pp. 637-638.

Eliot E., 2016, « Une approche spatiale des instruments de l'action publique : l'exemple des réformes du secteur de la santé en Inde » In Annales de géographie , 2, 708, pp. 193-212.

Fleuret S., 2006, Les associations dans le système sanitaire et social en France : les territoires de l'invisible. Economie et solidarités, pp.107-128.

Fleuret S., Skinner M., 2010, « Mieux comprendre le rôle de l'économie sociale dans les services sociaux et de santé : exemples choisis en France et au Canada ", Revue internationale de l'économie sociale, $\mathrm{n}^{\circ} 315$, février, pp. 75-89.

Fleuret S., 2016, « questionner la territorialisation de la santé en France » In Clavez M., Hardy A-

C. (Eds), Santé et territoires. Des soins de proximité aux risques environnementaux, Presses universitaires de Rennes

Frederiksson M., Blomqvist P., Windlad, U., 2012, « Conflict and Compliance in Swedish Health Care Governance: Soft Law in the 'Shadow of Hierarchy' » in Scandinavian Political Studies, 35, 1, pp 48-70.

Giaimo S., Manow P., 1999, « Adapting the Welfare State: The Case of Health Care Reform in Britain, Germany, and the United States » in Comparative Political Studies, 328

Gravier J-F., 1947, Paris et le désert français , Le Portulan. Rééditions Flammarion 1953, 1958, 1972.

Guilbaud A., 2015, "Les partenariats public-privé sanitaires internationaux : diffusion et incarnation d'une norme de coopération" in Mondes en développement, 2, 170, pp. 91-104.

Halpern C., Lascoumes P., Le Gales P., dir., 2014, L'instrumentation de l'action publique. Controverse, résistance, effets, Presses de Sciences Po, Paris, $520 \mathrm{p}$.

Henrard J-C., 1995, Plans et planification définition et histoire, Actualité et Dossier en Santé Publique, $\mathrm{n}^{\circ} 11, \mathrm{p} . \mathrm{II}-\mathrm{V}$. 
Hughes D., Leethongdee S., Osiri S., 2010, « Using economic levers to change behaviour: The case of Thailand's universal coverage health care reforms » in Social Science \& Medicine, 70, pp.447-454.

Kada N., 2012, La réforme de l'Etat territorial, Revue française d'administration publique, 2012/1, n¹41, p. 109-120.

Lane H., Sarkies M., Martin J., Haines T., 2017, «Equity in healthcare resource allocation decision making: a systematic review ", in Social Science and Medicine, 17, pp. 11-27.

Lascoumes P., Simard L., 2011, « L'action publique au prisme de ses instruments. Introduction », Revue française de science politique, 1, 61, p. 5-22.

Le Lidec P., 2011, Décentralisation, in Pasquier R, Guigner S. et Cole A. (dir.), Dictionnaire des politiques territoriales, Paris : Les Presses de Science Po, pp. 127-139.

Lewis J.M., Baeza J.L., Alexander D., 2008, « Partnerships in primary care in Australia: network structure, dynamics and sustainability » in Social Science and Medicine, 67, 2, pp. 280-91.

Measson L., 2008, L'apport des territoires de projet à la géographie politique, HAL [en ligne], Avril 2008, consulté le 5 juillet 2016. https://hal.archives-ouvertes.fr/halshs-00291708/document .

Moine A., 2006, «Le territoire comme un système complexe : un concept opératoire pour l'aménagement et la géographie », L’Espace Géographique, Tome 35, 2006/2, pp. 115-132.

Monneraud L., 2012, Collectivités, territoires et santé : regards croisés sur les frontières de la santé, Colloque GRALE-CERAPS-Université Lille 2. Murray S.F., Bisht R., Pitchforth E., « Emplacing India's "medicities" ", in Health and Place, 42, 2016, pp. 69-78

Nahapetian N., 2012, «Action publique : état d'alerte pour les associations », Alternatives économiques, 11/2012 ( ${ }^{\circ}$ 318), p. 38-38.

Parnaix A. et Zugetta I., 2015, De l'intercommunalité aux coopérations souples, Les Cahiers de l'IAU, 172, pp. 24-28.

Pollock A., Price D., 2014, « PFI and the National Health Service in England », http:// www.allysonpollock.com/wp-con-tent/uploads/2013/09/AP_2013_Pollock_PFINHSEngland.pdf (consulté en décembre 2016).

Polton D., 2004, Décentralisation des systèmes de santé : un éclairage international, RFAS, 2004/4, vol. 4, pp. 267-99.

Prince R., Kearns R., Craig D., 2006, « Governmentality, discourse and space in the New Zealand health care system, 1991-2003 ", in Health and Place, 12, 3, pp. 253-66.

Rajasekhar D., Berg E., Ghatak M, Manjula R., Roy S., 2011, « Implementing Health Insurance: The Rollout of Rashtriya Swasthya Bima Yojana in Karnataka » in Economic and Political Weekly, 46, 20, pp.56-63.

Raman V., Björkman J., 2015, Public-Private Partnerships in healthcare, in Kuhlmann E., Blank R., Bourgeault I., Wends C ; (Ed), The Palgrave International Handbook of Healthcare Policy and Governance, Palgrave Macmillan UK, pp. 376-92.

Rican S., Vaillant Z, 2009, «Territoires et santé : enjeux sanitaires de la territorialisation et enjeux territoriaux des politiques de santé. Commentaire ", Sciences sociales et santé , 1, 27, p. 33-42.

Sabi J., 2106, Une analyse théorique de la coordination dans le domaine des soins : application aux systèmes de soins coordonnés, in Santé Publique, 2, 28, pp. 223-234. 
Schapman-Segalie S., Lombrail P., 2013, Etat et collectivités territoriales : la contractualisation et les contrats locaux de santé, in Collectivités territoriales et santé, ADSP n ${ }^{\circ}$ 82, pp 44-46.

Soura B.D., Coulibaly S.S., 2014, « Analyse du processus de décentralisation des services de santé en Côte d'Ivoire » in Médecine et Santé Tropicales, vol. 24, pp. 151-6.

Torre A., 2015, Gouvernance territoriale et conflits d'usages, WikiTerritorial du CNFPT [en ligne], consulté le 30 juin 2016. http://www.wikiterritorial.cnfpt.fr/xwiki/wiki/econnaissances/view/ Notions-Cles/Gouvernanceterritorialeetconflitsdusages .

Vaguet A., 2011, « La santé en Territoires ", Actes du colloque international du CIST, Fonder les sciences du territoire, Paris, p. 461-165.

Vanier M., 2015, Démocratie locale : changement d'horizon ?, in Esprit, 2015/2, p. 109-118.

Vicente R., Castillejo J., 2012, «The role of Public Private Partnerships : The Brazilian experience of modernizing hospitals in Sao Paulo Prefecture Health Secretariat » in World Hospitals and Health Services, Vol. 48, 2, pp. 20-4.

Wilensky, G., 2012, G. « Re-engineering health systems: the U.S. Experience », in Social Science and Medicine 74, 5, pp. 674-6.

\section{NOTES}

1. Pour une définition, voir par exemple Chapoz, Pupion, 2012.

2. Par exemple, Castillejo V.R., 2012.

3. The Private Finance Initiative (PFI) au Royaume-Uni depuis le début des années 1990.

4. http://www.pbnrhm.org/docs/mission_doc.pdf (consulté en décembre 2016).

5. Cette recherche d'articulation est dans les faits très complexes : en témoigne, à titre d'exemple, un récent rapport de la délégation aux collectivités territoriales du Sénat de mai 2016. Il fait état d'une distorsion entre la profusion d'instances de dialogues (près de 500) et le ressenti des élus locaux qui éprouvent des difficultés à être écoutés et à peser réellement sur les décisions.

\section{RÉSUMÉS}

Ces dernières décennies, les transformations des systèmes de santé dans le monde se traduisent par des processus de territorialisation, sous-tendus par trois tendances principales : la régulation financière, le développement des partenariats et le transfert des compétences.

Dans ce papier, nous resituons tout d'abord le processus de territorialisation dans le cadre plus général des questions de décentralisation, de gouvernance et de gouvernement. En se focalisant sur le cas français, nous analysons ensuite l'articulation entre la décentralisation dite fonctionnelle en santé et les processus plus généraux de décentralisation des institutions. Pour conclure, nous questionnons les enjeux opérationnels et organisationnels qui en découlent. 
In this paper, we focus on the common trends of transformations of health care systems in the world. Based on the identification of three logics that root these evolutions (financial regulation, development of cooperation and skill/power transfers), we define territorialization as a process. It refers to projects of planning for which actors implement strategies to produce controlled and bounded spaces. In this process, scales, distances enacted through instruments are involved for putting public policy into practice and space. These actions give rise to different forms of territorialization in which different modes of governance, government and uses are embedded.

In a second part, we focus on the case of France and the ways instruments have been used to develop public policies. We insist on the disconnections between the political and administrative decentralization with the evolution of the health sector. The general trend of decentralization of French administration during the eighties is characterized by a greater proximity of public decision with citizen, which involves more action for local authorities. A structured vision of decentralization appears only at the beginning of 2010s based on potential differentiation of standard on the territory and the possibility for regions to provide guidance to others local authorities. During these periods, health sector remains on the sidelines of the legislative evolution. Although regional level was defined as the structuring level of organization for health care in the hospital law of 1991, regionalization of public health policy really operated in 2009 with the Patients, Health and Territories Law (HPST) creating regional health agencies. These ones, with the legal personality are a type of functional decentralization, which are submitted to state monitoring. Concentrating the decision-making power at the regional level and breaking down barriers between sectoral policies, this law simplified local organization of health sector governance. It is operated in a regional health plan which relies on contractual partnerships with a lot of actors including local authorities which became a major actor to decline the plan at the local level.

In a third part, we discuss the stakes, options and problems that actors have to face in the current stage of decentralization. Combined with deconcentration of others services, overlapping, cross-financing occurred and involved lower clarity of administrative organization. Two major stakes are identified. Firstly, the capabilities for actors to form a wide coalition sharing objectives and keeping up with changes in stakeholder's dynamics, like political changeovers and budget cuts. Secondly, a better coordination of actors, in order to create favorable conditions for cooperation and complementarities.

INDEX

Mots-clés : territorialisation, décentralisation, systèmes de santé, régulation financière, partenariat, transfert de compétences

\section{AUTEURS}

\section{EMMANUEL ELIOT}

Professeur, Université de Rouen, France

\section{VÉRONIQUE LUCAS-GABRIELLI}

Directrice de recherche, Irdes, Paris, France 


\section{CATHERINE MANGENEY}

Démographe-urbaniste à l'Institut d'aménagement et d'urbanisme de la région Île-de-France (IAU-ÎdF) 